\title{
STUDIES ON CONTROL OF VISCERAL LEISHMANIASIS: IMPACT OF DOG CONTROL ON CANINE AND HUMAN VISCERAL LEISHMANIASIS IN JACOBINA, BAHIA, BRAZIL
}

\author{
DAVID A. ASHFORD, JOHN R. DAVID, MIRALBA FREIRE, ROBERTA DAVID, ITALO SHERLOCK, \\ MARIA DA CONCEIÇÃO EULÁLIO, DIANA PEDRAL SAMPAIO, AND ROBERTO BADARO \\ Department of Tropical Public Health, Harvard School of Public Health, Boston, Massachusetts; Universidade Federal da Bahia, \\ Salvador, Brazil; Fundacao Oswaldo Cruz-Bahia, Salvador, Brazil; Cornell University Medical College, New York, New York
}

\begin{abstract}
To assess the effect of removing leishmania-infected dogs on the incidence of visceral leishmaniasis, a controlled intervention study was performed in northeast Brazil. The attempted elimination of seropositive dogs resulted in an initial significant decrease in the annual incidence of seroconversion among dogs from $36 \%$ to $6 \%$ over the first two years. In the following two years, the incidence increased to $11 \%$ and $14 \%$, respectively. In a control area in which dogs were surveyed but seropositive dogs were not removed, the cumulative incidence did not vary significantly from year to year, ranging from $16 \%$ to $27 \%$. In the intervention area, the prevalence of dog seropositivity decreased from $36 \%$ before the intervention to $10 \%$ and remained stable. These findings suggest that attempting to remove seropositive dogs is insufficient as a measure for eradicating visceral leishmaniasis in dogs. However, the force of transmission of infection among dogs can be reduced by such programs. Also, when the number of human cases before and after the start of the intervention was calculated, a significant decrease in incidence of disease in the intervention area was observed among children less than 15 years of age $(P<0.01)$. The results of this intervention study suggest that the elimination of the majority of seropositive dogs may affect the cumulative incidence of seroconversion in dogs temporarily and may also diminish the incidence of human cases of visceral leishmaniasis.
\end{abstract}

Following the discovery of canine visceral leishmaniasis in Tunisia by Nicolle and Conte in 1908, dogs have been implicated as important reservoirs for visceral leishmaniasis. ${ }^{1}$ Adler and Theodore described in detail in the 1920 s and 1930s the coprevalence and similarity of disease in human and dog populations in the Mediterranean. ${ }^{2}$ They were the first to conclude that Leishmania tropica was the causative organism of cutaneous leishmaniasis in both dogs and humans. These findings suggested that dogs might serve as a reservoir for human leishmaniasis, cutaneous or visceral.

Since these early findings, several studies have implicated the involvement of dogs in transmission of visceral leishmaniasis. ${ }^{3-5}$ These studies have described the presence of canine seropositivity in areas of endemic kala-azar. However, evidence of infection within two hosts does not establish a causal relationship between them.

As a result of this literature, control programs for visceral leishmaniasis often include elimination or treatment of infected dogs. Control programs also can include treatment of human cases, sand fly vector control, or elimination of other suspected animal reservoirs. In areas of Brazil in which visceral leishmaniasis is endemic, control programs emphasize serologic surveys of dogs and humans, and killing of seropositive animals. However, to date no study has conclusively shown that this strategy is effective.

Between June 1989 and May 1993, a controlled intervention study was conducted to determine whether the elimination of seropositive dogs would decrease the incidence and prevalence of visceral leishmaniasis in dogs and in humans. This report summarizes the effects of the dog control on canine infection as determined by serologic testing. Initial findings regarding the incidence of pediatric cases are also presented.

\section{METHODS}

Study area. The study area, Jacobina, Bahia, Brazil, has been previously described. ${ }^{6,7}$ Briefly, the city of 40,000 in- habitants lies $350 \mathrm{~km}$ from the coastal city of Salvador. The endemic foci of visceral leishmaniasis are located at the periphery of the city.

The areas of intervention and control were two neighborhoods at the edge of the city separated by a river (approximately 50 meters wide) and a distance of $4 \mathrm{~km}$. These areas were chosen because a longitudinal study showed that visceral leishmaniasis had been transmitted there continuously during the last 10 years. The incidence of kala-azar in children less than 15 years old has averaged six cases/1,000/ year. For both areas, altitude, socioeconomic status of the population, and population demographics were similar.

Dog population. The dog population was composed mainly of mongrels with little access to veterinary care. The dog population, in both control and intervention areas, had a mean age of 2.5 years.

Dog survey. Between 1989 and 1993, an annual census of domestic dogs in both areas was completed; blood samples were collected on all available dogs. Basic descriptive information was collected for each dog. Clinical signs were recorded and will be reported elsewhere. After informed consent was obtained from the owners, the dogs were bled from the cephalic vein and vaccinated for rabies if needed.

Serology. Serologic tests were performed using the Falcon Assay Screening Test-enzyme-linked immunosorbent assay (FAST-ELISA ${ }^{\circledR}$; Becton Dickinson, Oxnard, CA). The FAST-ELISA has been shown to have a sensitivity and specificity equal to standard ELISA and superior to immunofluorescence assay. ${ }^{7}$ Blood was collected in 1-ml sterile tubes containing citrate; plasma was evaluated by FAST-ELISA the same day at the central health post or stored at $-20^{\circ} \mathrm{C}$ for 2-4 weeks until testing. Dogs with positive serologic results were retested and in many cases evaluated by bone marrow aspiration under ketamine/xylazine anesthesia for parasitologic confirmation. Bone marrow samples were eval- 


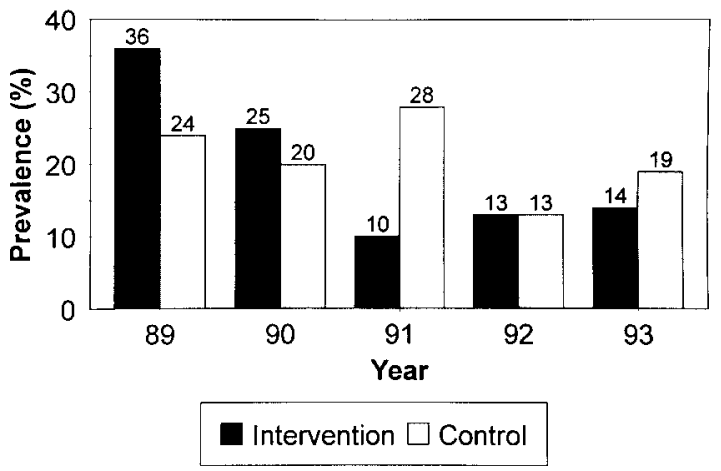

FIGURE 1. Prevalence of seropositivity in the dogs of the control and intervention areas.

uated by hamster or culture inoculation as previously described. ${ }^{7}$

Intervention. In the intervention area, and following informed consent, national public health service personnel attempted to remove all seropositive dogs to a veterinary public health post where they were anesthetized (as described above) and killed using intravenous potassium oxalate. Dog removal was begun in 1989, following the first serologicsurvey. In the control area, dogs were not removed by the investigating team or public health officials. No vector control programs occurred during the study period in Jacobina.

Pediatric cases. A case was defined as a child less than 15 years old with parasitologically confirmed visceral leishmaniasis between January 1986 and December 1993. Children with histories of previously confirmed visceral leishmaniasis were not included. Cases were identified using all available records from all health care centers in Jacobina.

The ethical review process for the portion of the project involving humans was conducted by the Committee for the Use of Human Subjects of the Harvard School of Public Health, the Commisâo de Etica of the Hospital Edgar Santos, The Universidade Federal da Bahia, and the Centro de Pesquisas Goncalo Muniz, FIOCRUZ (Salvador, Brazil). No personal identifiers were obtained during the health care center record review.

Statistical methods. Annual cumulative incidences for 1990-1993 were calculated and changes in prevalence and incidence were compared using the chi-square test and chisquare test for trend across time. Cumulative incidence was calculated as the number of dogs that seroconverted over the number of dogs present that were negative in the previous serologic-survey. To compare the change in incidence between the intervention and the control area, we used Poisson regression and the Statistical Package on Interactive Data Analysis software (Macquaire University, Sydney, Australia). ${ }^{8}$

\section{RESULTS}

The seroprevalence among dogs for June 1989 through March 1993 (the study period) in the intervention and control areas is presented in Figure 1. The prevalence decreased from $36 \%$ in 1989 to $10 \%$ in 1991, and remained without significant change for the next two years despite the immigration of new positive dogs. On the other hand, the prev-

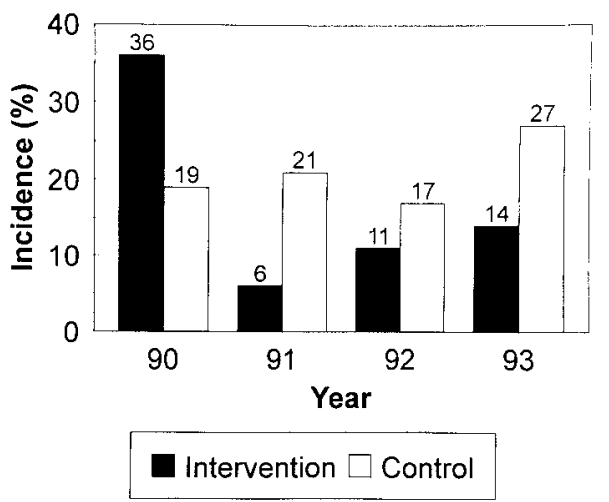

FIGURE 2. Cumulative incidence of seroconversion for Leishmania chagasi infection among dogs in the control and intervention areas.

alence in the control area did not change significantly over the study period, although a decrease to $13 \%$ was observed in 1992.

The cumulative incidences of L. chagasi infection (as determined by serology) for the intervention and control areas are shown in Figure 2. In the intervention area, the incidence of seroconversion decreased significantly in the first years of the study from $36 \%$ to $6 \%(P<0.001)$, and remained low with no significant change over the next three years.

For the control area, there was no significant change in incidence. Five (19\%) of 26 seronegative dogs followed from 1989 were found to have seroconverted by 1990. Seven (26\%) of 34 seronegative dogs from 1990 that were bled in 1991 were seropositive. In 1992, four (17\%) of 23 dogs had seroconverted. In 1993, six (27\%) of 22 dogs available had seroconverted.

In comparing the incidence of infection among dogs between the intervention and control areas, the difference was not significant for 1990. In 1991, the incidence of $6 \%$ in the intervention was significantly lower than the incidence of $21 \%$ in the control area $(P<0.05)$. In 1992 and 1993, the incidences for the control and intervention areas were not significantly different. Furthermore, the observed decrease in incidence in the intervention area during the four years was not significantly different from the observed incidence in the control area over the four years of study $(P=0.07)$.

The data for the dog surveys in the intervention area are shown in Table 1. To facilitate understanding of this table, the first year is described here in detail. In 1989, of the 235 dogs studied in the intervention area, 84 were positive (36\%). Of these, 58 (69\%) were killed, died, or moved to another area. In 1990, 26 of the original 84 seropositive dogs were still present and seven of them had become seronegative. Twenty of the 151 seronegative dogs had converted to seropositive; 36 were still seronegative, while 79 had died or moved and 16 were not bled. The incidence was calculated by dividing the number of dogs that seroconverted by the number that were available for survey (seronegative plus seropositive). Thus, in 1990, the incidence was 20 divided by 56 , or $36 \%$. In 1990, 61 dogs were seropositive, which included 22 new dogs. One hundred eighty-seven were seronegative, which included 144 new dogs.

Pediatric cases/1,000, as defined above, for the interven- 
TABLE 1

Summary of canine surveys in the intervention area, Jacobina, Bahia, Brazil, 1989-1993*

\begin{tabular}{|c|c|c|c|c|c|c|c|}
\hline \multicolumn{2}{|c|}{ Annual summary } & \multicolumn{6}{|c|}{ Outcome for the follow-up year } \\
\hline & No. $(\%)$ & $\begin{array}{c}\text { Sero- } \\
\text { positive }\end{array}$ & $\begin{array}{c}\text { Sero- } \\
\text { negative }\end{array}$ & Removed & Died & Moved & $\begin{array}{l}\text { Not } \\
\text { bled }\end{array}$ \\
\hline \multicolumn{8}{|c|}{ 1989-1990 } \\
\hline Pos & $84(36)$ & 19 & 7 & \multirow[t]{3}{*}{35} & 14 & 9 & 0 \\
\hline $\mathrm{Neg}$ & 151 & 20 & 36 & & 47 & 32 & 16 \\
\hline Total & 235 & $39 \dagger$ & $43 \ddagger$ & & & & \\
\hline \multicolumn{8}{|c|}{ 1990-1991 } \\
\hline Pos & $61(25)$ & 3 & 4 & \multirow[t]{3}{*}{32} & 8 & 7 & 7 \\
\hline $\mathrm{Neg}$ & 187 & 3 & 52 & & 62 & 43 & 27 \\
\hline Total & 248 & $6 \dagger$ & $56 \ddagger$ & & & & \\
\hline \multicolumn{8}{|c|}{ 1991-1992 } \\
\hline Pos & $7(10)$ & 2 & 2 & \multirow[t]{3}{*}{3} & & & \\
\hline Neg & 63 & 4 & 31 & & ? & ? & 27 \\
\hline Total & 70 & $6 \dagger$ & $33 \ddagger$ & & & & \\
\hline \multicolumn{8}{|c|}{ 1992-1993 } \\
\hline Pos & $15(13)$ & 1 & & \multirow[t]{3}{*}{11} & 1 & 1 & 1 \\
\hline Neg & 116 & 8 & 50 & & 15 & 33 & 10 \\
\hline Total & 131 & $9 \dagger$ & $50 \ddagger$ & & & & \\
\hline \multicolumn{8}{|l|}{1993} \\
\hline Pos & $23(14)$ & & & & & & \\
\hline Neg & 141 & & & & & & \\
\hline Total & 164 & & & & & & \\
\hline
\end{tabular}

* Positive (Pos) equals total number of seropositive dogs in follow-up group $(\dagger)$ plus new dogs that have moved into the area that were seropositive. Negative (Neg) equals total number of seronegative dogs in follow-up group (‡) plus new dogs that have moved into the area that were seronegative.

tion and control areas are shown in Table 2. In the intervention area, the observed decrease in the number of pediatric cases following the initiation of the intervention, was significantly different from the control area $(P<0.01)$ using Poisson regression and considering each year's data. A collapsed version of the data for the four years before and after the intervention is shown in Table 3. The decrease observed in the intervention area was significantly greater than the increase seen in the control area when the data are collapsed $\left(\chi^{2}=20.72, P=0.000005\right)$.

\section{DISCUSSION}

In this study, the attempt to remove seropositive dogs was associated with a decrease in the incidence of visceral leishmaniasis in dogs, although transmission was not eliminated. Furthermore, a significant decrease in the incidence of human pediatric cases of visceral leishmaniasis was observed in the area of intervention.

Over a four-year period, this controlled intervention study resulted in a significant decrease in the cumulative incidence of $\operatorname{dog}$ seroconversion from $36 \%$ to $14 \%(P<0.05)$ despite the continued presence of some infected dogs. Although the overall change in incidence within the intervention area was significant, when the decrease was compared with the control area there was no significant difference in observed incidence between the intervention and control areas over the entire period of study $(P=0.07)$. One of the limitations of the study was the small samples of dogs available from year to year. The $P$ value of 0.07 suggests that there may have been a trend toward decreasing infection in dogs in the in-
TABLE 2

Numbers of pediatric* incident cases/1,000 inhabitants/year for the intervention and control areas (the intervention was initiated in the second half of 1989)

\begin{tabular}{lccrrrrrr}
\hline & 1986 & 1987 & 1988 & 1989 & 1990 & 1991 & 1992 & 1993 \\
\hline Intervention & 12 & 5 & 14 & 10 & 3 & 2 & 2 & 2 \\
Control & 6 & 2 & 2 & 12 & 8 & 12 & 4 & 2 \\
$\quad$ Total & 18 & 7 & 16 & 22 & 11 & 14 & 6 & 4 \\
\hline * Pediatric cases are defined as parasitologically confirmed visceral leishmaniasis in chil- \\
dren less than 15 years old. Values are cases are per 1,000 inhabitants.
\end{tabular}

tervention area; however, the sample size limitations (partly due to a loss to follow up) may have influenced our ability to detect that decrease. Despite initiating dog removal after the first survey in 1989, we found an incidence of $36 \%$ in the intervention area in 1990. Possible reasons for this observation might be increased force of transmission due to reduction in the dog population or delays in dog removal.

The cumulative incidence was significantly different between the intervention and control areas in the second year of the intervention $(P<0.05)$. After that year, 1991, we did not detect a significant difference in incidence when comparing the intervention and control areas. This could be due to sample size limitations. Also, the transmission of infection was not interrupted since newly infected dogs were detected each year of the study.

One possible explanation for continued transmission involves the efficiency and timing of the removal of dogs. Dye emphasized two important variables in the dynamic of visceral leishmaniasis transmission: the seasonality of the sand fly population and the number of infected reservoir animals. ${ }^{9}$ $\mathrm{He}$ suggested that it might be important to eliminate dogs before the sand fly population peaks. We were unable to do this in our study.

Another explanation for the continued incidence is the presence of reservoirs other than dogs. Visceral leishmaniasis is associated with several mammalian species in endemic disease areas. Domestic dogs and foxes (Cerdocyon thous and Lycalopex vetulus) are thought to be the most important source of organisms for sand fly infection in South America. ${ }^{3}$ However, the South American opossums (Didelphis albiventrus and D. marsupialis) have also been found to be infected at rates equal to or greater than dogs. ${ }^{10}$ Rattus rattus is an important reservoir of $L$. infantum in the Middle East, although it has not been shown to be important in our area. In reservoir studies in Jacobina, $D$. albiventris was the most frequently captured mammal with an L. chagasi infection prevalence of $5 \% .{ }^{11}$ Currently, it is thought that the sylvatic cycle between foxes and other mammals results in peridomestic transmission when domestic dogs are infected by the sand flies that have fed on roving sylvatic hosts near

TABLE 3

Number of pediatric incident cases/1,000 inhabitants before and after the intervention*

\begin{tabular}{lcc}
\hline & $1986-1989$ & $1990-1993$ \\
\hline Intervention area & 41 & 9 \\
Control area & 22 & 26
\end{tabular}

* The quadrant totals represent a sum of cases in the four-year periods seen in Table 2 . 
human dwellings. ${ }^{3}$ Human-to-human transmission of leishmaniasis via sand flies, as occurs in India, has also been suspected in Brazil. . $^{3,6}$

In Jacobina, there is a typical seasonal distribution of cases of human visceral leishmaniasis, with natural peaks of incidence occurring from October to February. ${ }^{6}$ During the period of this study, this pattern of human cases continued. The finding of a significant decrease in the average number of pediatric cases after the intervention suggests that the removal of infected animals may affect the transmission to humans. However, several other factors could account for this observed change. Study effects and seasonal cyclic variations cannot be ruled out; the increased intensity of research may have led the population to take other precautions against acquiring disease. Because there were more cases in the intervention area before the start of the study, there may have been fewer susceptible inhabitants in that area. Finally, the actual numbers of cases are small. The population of Jacobina has been well studied for 20 years, and it is unlikely that any patients were misclassified.

Dogs have been incriminated as an important reservoir of L. chagasi in endemic disease areas..,12,21 Previous control trials have suggested that removing seropositive dogs is important in the control of visceral leishmaniasis in humans. ${ }^{12}$ Of note, much of the evidence for a role of the dog as a reservoir is still based on studies involving coprevalence of disease. ${ }^{13-17}$ Adler and Theodore pointed out as early as 1929 that coprevalence alone does not provide a convincing argument for direct transmission, and they suggested that human beings served as the reservoir for dogs. ${ }^{18}$ The prevalence of dog visceral leishmaniasis within the Mediterranean region has been reported to be from $1 \%$ to $42 \% .{ }^{19}$ The disease in humans is increasing in incidence in southern France and in Sicily. ${ }^{20}$ However, current knowledge of the role of domestic canines in transmission of $L$. donovani or $L$. chagasi remains incomplete.

Gradoni and others showed a decrease in incidence and prevalence of clinical canine leishmaniasis following a control program involving treatment of oligosymptomatic, asymptomatic, and symptomatic dogs with pentavalent antimonials. ${ }^{21}$ No control area was available in that study, and other studies have described high levels of recurrence and dormancy in treated animals, ${ }^{22}$ suggesting that treatment of dogs may add to the force of transmission in the long run. In China, an intensive control program including treatment of cases, use of insecticide, and killing of infected dogs eliminated the disease. ${ }^{23}$ However, in recent years the disease has re-emerged in dogs and humans. In Brazil, while certain studies have suggested there was a decrease in human prevalence of disease when seropositive dogs were removed, such control was combined with vector control and human case treatment. ${ }^{12,24}$ Therefore, it is difficult to attribute the observations to dog control, especially considering that vector control programs in Italy for sand fly fever and in India for malaria resulted in concurrent control of human visceral leishmaniasis.

During the period of our study, there was no programmed spraying or other vector control measures in Jacobina, Bahia. Also, in a parallel study, no significant variation in the sand fly population capture rates was seen over the four-year period (Sherlock I, unpublished data).
Despite the decrease in incidence observed in this study, important questions remain. Regarding the decrease in prevalence and incidence in the control area, a possible study effect could have occurred in which sick dogs may have been removed by owners. Also, the prevalence of visceral leishmaniasis was not identical in both areas before the intervention.

We experienced several difficulties in our study that might explain the continued incidence of disease. Seropositive dog removal was never completely efficient, despite the focus of the study. Also, basic questions remain regarding infection and seroconversion. Our study comparing polymerase chain reaction and serologic testing indicates that serologic screening underestimates infection prevalence..$^{25}$

The results of the above intervention study suggest that the elimination of the majority of seropositive dogs may affect the cumulative incidence of seroconversion in dogs temporarily and may also diminish the incidence of human cases of visceral leishmaniasis. However, continued transmission, despite dog removal, suggests several possibilities: that other reservoirs may be involved in maintaining canine infection, that it may be necessary to remove all seropositive dogs, or that serologic methods are inadequate for detecting all infected dogs that can transmit the disease to sand flies. Although a change in incidence of pediatric cases was observed, we cannot be certain that this was directly related to our dog control program. Regarding zoonotic disease control, interventions aimed at an individual species may be less effective where multiple reservoirs are involved in transmission. Further studies are needed to determine the influence of other potential reservoirs, to understand the course of infection and seroconversion in dogs, and to evaluate the effect of a more efficient removal of infected dogs on both canine and human visceral leishmaniasis. In summary, cost-effectiveness studies evaluating dog control programs are required before national control measures are recommended.

Acknowledgments: We thank Lori Hutwagner for statistical assistance, and Antônio Souza, Carlos Miranda, and the Fundação Nacional da Saúde for technical assistance.

Financial support: This work was supported in part by a grant from the NIH (AI-16305-13) and the Special Program for Research and Training in Tropical Diseases (TDR) (World Bank/UNDP/WHO).

Authors' addresses: David A. Ashford, Centers for Disease Control and Prevention, Mailstop C-09, Atlanta, GA 30333. John R. David and Roberta David, Department of Tropical Public Health, Harvard School of Public Health, Boston, MA 02115. Miralba Freire, Maria da Conceição Eulálio and Diana Pedral Sampaio, Universidade Federal da Bahia, Salvador, Brazil. Italo Sherlock, Fundacao Oswaldo Cruz-Bahia, Salvador, Brazil. Roberto Badaro, Universidade Federal da Bahia, Salvador, Brazil.

\section{REFERENCES}

1. Nicolle C, Comte M, 1908. Recherches sur la kala-azar enterprises a l'Institut Pasteur de Tunis, IV. Origine canine du kalaazar. Arch Inst Pasteur Tunis 3: 59-62.

2. Adler S, Theodore O, 1932. Investigations on Mediterranean kala azar. VI-canine visceral leishmaniasis. Proc $R$ Soc Lond 110: 402-412.

3. Shaw JJ, Lainson FRS, 1987. Ecology and epidemiology: New World. Peters W, Killick-Kendrick R, eds. The Leishmaniases in Biology and Medicine. Volume 1. London: Academic Press, 365 . 
4. Neogy AB, Vouldoukis I, Silva OA, Tselentis Y, Lascombe JC, Segalen T, Rzepka D, Monjour L, 1992. Serodiagnosis and screening of canine visceral leishmaniasis in an endemic area of Corsica: applicability of a direct agglutination test and immunoblot analysis. Am J Trop Med Hyg 47: 772-777.

5. Bettini S, Gradoni L, 1986. Canine leishmaniasis in the Mediterranean area and its implications for human leishmaniasis. Insect Sci Applicat 7: 241-245.

6. Badaro R, Jones TC, Lorenco R, Cerf BJ, Sampaio D, Carvalho EM, Rocha H, Teixera R, Johnson WD Jr, 1986. A prospective study of visceral leishmaniasis in an endemic area of Brazil. J Infect Dis 154: 639-649.

7. Ashford DA, Badaro R, Eulalio C, Freire M, Miranda C, Zalis MG, David JR, 1993. Studies on the control of visceral leishmaniasis: validation of the Falcon assay screening test-enzyme-linked immunosorbent assay (FAST-ELISA ${ }^{\circledR}$ ) for field diagnosis of canine visceral leishmaniasis. Am J Trop Med Hyg 48: 1-8.

8. Dawson-Saunders B, Trapp R, 1990. Basic and Clinical Biostatistics. New York: Appleton \& Lang Publishers, 154-156.

9. Dye C, 1992. Leishmaniasis epidemiology: the theory catches up. Parasitology 104: S7-S18.

10. Corredor A, Gallegro JF, Tesh RB, Morales A, Ferro de Carrasquilla C, Young DG, Kreutzer JB, Palau MT, Caceres E, Peleaz D, 1989. Didelphis marsupialis, an apparent wild reservoir in Columbia, South America. Trans R Soc Trop Med Hyg 83: 195-197.

11. Sherlock IA, 1984. Natural infection of the opossum Didelphis albiventris (Marsupialia, Didelphidae) with Leishmania donovani, in Brazil. Mem Inst Oswaldo Cruz 79: 511-512.

12. Alencar JE, 1961. Profilaxia do calazar no Ceara, Brazil. Rev Inst Med Trop Sao Paulo 3: 175-180.

13. Marty P, Le Fichoux I, Giordana D, Brugnetti A, 1992. Leishmanin reaction in the human population of a highly endemic focus of canine leishmaniasis in Alpes-Maritimes, France. Trans $R$ Soc Trop Med Hyg 86: 249-250.

14. Mancianti F, Gradoni L, Gramiccia M, Pieri S, Marconcini A,
1986. Canine leishmaniasis in the Isle of Elba, Italy. Trop Med Parasitol 37: 110-112.

15. Mutinga MJ, Ngoka JM, 1980. The isolation of leishmanial parasites from domestic dogs in the Machakos District of Kenya, and the possible role of dogs as reservoirs of kala-azar in East Africa. Ann Trop Med Parasitol 74: 139-144.

16. Corredor A, Gallego JF, Tesh RB, Morales A, Ferro de Carrasquilla C, Young DG, Kreutzer JB, Palau MT, Caceres E, Peleaz D, 1989. Epidemiology of visceral leishmaniasis in Columbia. Am J Trop Med Hyg 40: 480-486.

17. Lanotte G, Rioux JA, Croset H, Vollhardt Y, 1978. Ecolgie des Leishmanisoses dans le sud de la France. 9. Les methodes d'echantillonnage dans le depistage et l'analyse de l'enzootie canine. Ann Parasitol 53: 33-45.

18. Adler S, Theodore O, 1929. Attempts to transmit Leishmania tropica by bite; transmission of L. tropica by Plebotomus sergenti. Ann Trop Med Parasitol 23: 1-18.

19. Mancianti F, Meciani N, 1988. Specific serodiagnosis of canine leishmaniasis by indirect immunofluorescence, indirect hemagglutination, and counterimmunoelectrophoresis. Am J Vet Res 49: 1409-1411.

20. Ashford RW, Bettini S, 1987. Ecology and epidemiology: Old World. Peters W, Killick-Kendrick R, eds. The Leishmaniase in Biology and Medicine. Volume 1. London: Academic Press, 291.

21. Gradoni L, Gramiccia M, Mancianti F, Pieri S, 1988. Studies on canine leishmaniasis control. 2. Effectiveness of control measures against canine leishmaniasis in the Isle of Elba, Italy. Trans $R$ Soc Trop Med Hyg 82: 568-571.

22. Slappendel RJ, 1988. Canine leishmaniasis. A review based on 95 cases in the Netherlands. Vet $Q$ 10: $1-16$.

23. Leng YJ, 1982. A review of kala-azar in China from 1949-1959. Trans R Soc Trop Med Hyg 76: 531-537.

24. Sherlock IA, 1969. Observações sobre calazar em Jacobina - I - Historico e dados preliminares(*). Rev Bras Mal Doen Trop 14: 523-534.

25. Ashford DA, Bozza M, Miranda JC, Eulalio C, Freire M, Miranda C, Zalis MG, David JR, 1995. Comparison of the polymerase chain reaction and serology for the detection of canine visceral leishmaniasis. Am J Trop Med Hyg 53: 251-255. 